\title{
Correlação entre o topógr afo de disco óptico (TOPSS) e índices do campo visual
}

\author{
Cor relation betw een the topographic scanning system (TOPSS)and visual field in dices
}

Leopoldo Magacho ${ }^{1}$

Ana Maria Marcondes ${ }^{2}$

Vital Paulino Costa ${ }^{3}$

\section{RESUMO}

Objetivos: Avaliar a correlação entre os parâmetros do Topógrafo de Disco Óptico (TOPSS) e índices da perimetria computadorizada. Métodos: Cento e doze pacientes com glaucoma primário de ângulo aberto e 88 indivíduos normais foram incluídos no estudo. Todos os indivíduos foram submetidos ao exame oftalmológico completo, perimetria computadorizada (Humphrey 24-2, Full Threshold), avaliando-se Mean Deviation (MD) e Correct Pattern Standard Deviation (CPSD) e exame com TOPSS (average disc diameter, total disc area, cup area, cup shape, cup volume, average cup depth, average disc depth, neuroretinal rim (NRR) volume, NRR area, cup/disc area ratio, horizontal cup/disc ratio e vertical cup/disc ratio). A comparação entre os grupos foi realizada utilizando-se o Teste U de Mann-Whitney ou o Teste T de Student Independente. A correlação entre cada parâmetro do TOPSS e os índices do campo visual foi verificada utilizando-se a correlação de Spearman. Resultados: Os parâmetros do TOPSS que apresentaram as correlações mais significativas com os índices de campo visual foram: Cup Area(MD: $r=-0,538, p<0,001$; CPSD: $r=0,512, p<0,001$ ), VerticalC/DRatio (MD: $r=-0,506, p<0,001 ; C P S D: r=0,483, p<0,001)$ e Cup/Disc Area Ratio (MD: $r=-0,458, p<0,001 ;$ CPSD: $r=0,453, p<0,001$ ). Entretanto, houve importante variabilidade desses parâmetros em diferentes níveis de dano glaucomatoso, representados pelo MD e CPSD. Conclusão: As variáveis do TOPSS apresentam, no geral, correlação satisfatória com os índices do campo visual. Apesar de alguns desses parâmetros apresentarem correlação altamente significativa, existe grande variabilidade dos parâmetros estruturais para um mesmo nível de dano funcional, limitando a utilização isolada de parâmetros estruturais derivados do TOPSS no diagnóstico do glaucoma.

Descritores: Glaucoma/diagnóstico; Disco óptico/patologia; Perimetria; Técnicas de diagnóstico oftalmológico

\section{INTR ODUÇÃ O}

Nos últimos anos, têm-se verificado um crescente aumento no número de instrumentos de auxílio diagnóstico em oftalmologia. Em se tratando do glaucoma, novas máquinas têm apresentado a possibilidade de possível diagnóstico precoce da doença, antes mesmo da detecção de lesões no campo visual. A iniciativa tem surgido sobretudo pelo fato de que a lesão perimétrica ocorre em fases relativamente tardias do glaucoma, onde um número considerável de células ganglionares retinianas já foram lesadas (de $25-35 \%{ }^{(1)}$ a $\left.40 \%\right)^{(2)}$. Decorre também da demonstração de que o dano às estruturas do disco óptico ${ }^{(3-4)}$ e camada de fibras nervosas da retina ${ }^{(4-5)}$ podem se antecipar à perda de campo visual. 
Entre esses novos métodos de imagem, destacam-se a análise da camada de fibras nervosas da retina por meio da Polarimetria de Varredura a Laser (GDx, Laser Diagnostic Technologies, San Diego, CA, USA) ${ }^{(6-7)}$, e a topografia de disco óptico, realizada pelo Tomógrafo Retiniano de Heidelberg (HRT, Heidelberg Engineering, Heidelberg, Germany) ${ }^{(8-9)}$ ou o Topógrafo de Disco Óptico (TOPSS, Laser Diagnostic Technologies, San Diego, CA, USA) ${ }^{(10-11)}$. As informações estruturais provenientes dos instrumentos relacionados acima podem ser validadas através da comparação com métodos diagnósticos considerados como "gold standard" em glaucoma. Apesar de GDx, HRT e TOPSS avaliarem estruturalmente o dano glaucomatoso e o campo visual ser um exame funcional, é esperado que apresentem correlação, especialmente nos estágios mais avançados da doença. Estudos anteriores já testaram a correlação separadamente entre o $\mathrm{GDx}^{(12-13)}$, o topógrafo de disco óptico ${ }^{(14-17)}$ e o campo visual, sugerindo que determinados parâmetros dos instrumentos acima podem ser usados para quantificar o dano glaucomatoso.

Apesar do TOPSS e HRT usarem o mesmo princípio na aquisição das imagens (Sistema de Varredura Confocal), existem diferenças quanto às variáveis testadas em cada apare1 ho ${ }^{(11,18)}$, e na forma de delinear o disco óptico para proceder a análise. Portanto, as informações obtidas com o HRT não podem ser extrapoladas e utilizadas como premissas para o TOPSS. Poucos são os estudos que correlacionaram os parâmetros do TOPSS com os índices da perimetria computadoriza$\mathrm{da}^{(14-15)}$, sendo que apenas um teve como referência o campo visual Humphrey ${ }^{(14)}$. Esse estudo tem como objetivo testar a correlação entre os parâmetros do TOPSS e os índices do campo visual computadorizado.

\section{MÉTODOS}

Esse foi um estudo prospectivo comparativo. Após aprovação do Comitê de Ética da Universidade de Campinas (UNICAMP), todos os indivíduos selecionados foram submetidos a exame oftalmológico completo, que incluiu biomicroscopia detalhada, tonometria de aplanação (Goldmann), biomicroscopia de fundo de olho (retina e disco óptico) após midríase, campimetria computadorizada (Humphrey Visual Field Analyser II, programa 24-2, estratégia full threshold, Humphrey Systems, Dublin, CA, USA) e topografia de disco óptico (TOPSS). Apenas um olho por paciente foi aleatoriamente escolhido se ambos satisfizessem os critérios de inclusão.

Os critérios de inclusão para ambos os grupos foram: acuidade visual $\geq 20 / 30$, equivalente esférico $\leq 5$ dioptrias, diâmetro pupilar acima de $2 \mathrm{~mm}$ e dois exames confiáveis de campo visual (perda de fixação $<20 \%$, e falso positivos e negativos $<33 \%)^{(19)}$. Os critérios de exclusão (ambos os grupos) incluíram: história de qualquer doença sistêmica ou ocular (exceto glaucoma) que poderia causar alterações no exame de perimetria computadorizada ou TOPSS, cirurgia refrativa, olhos afácicos ou pseudofácicos e catarata importante ${ }^{(20)}$, que pudesse interferir nos exames de campo visual ou de imagem.
Os indivíduos normais foram recrutados entre os membros da Universidade, voluntários da equipe médica, familiares e amigos dos pacientes. Esses foram excluídos se apresentassem pressão intra-ocular (Pio) acima de $21 \mathrm{mmHg}$, disco óptico suspeito (perda localizada da rima, hemorragia de disco óptico, assimetria de escavação >0,2) ou campo visual alterado (definido a seguir).

Pacientes com glaucoma foram recrutados do ambulatório da especialidade da UNICAMP. Os critérios de inclusão para esse grupo foram: diagnóstico clínico de glaucoma primário de ângulo aberto (ou seja, duas ou mais medidas da Pio acima de $21 \mathrm{mmHg}$, gonioscopia demonstrando ângulo aberto, e lesão característica de disco óptico, definido como escavação aumentada, perda localizada da rima, hemorragia de disco óptico ou assimetria de escavação >0,2). Além disso, a presença de pelo menos dois campos visuais confiáveis com perda glaucomatosa característica, definido pelos critérios propostos por Hodapp-Parrish-Anderson, eram necessários ${ }^{(21)}$. Apenas o último campo visual foi considerado para efeito de análise.

Os pacientes glaucomatosos foram classificados pela severidade da lesão de campo visual de acordo com os seguintes critérios ${ }^{(7)}$ : a) dano inicial: Mean Deviation (MD) melhor ou igual que $-6 \mathrm{~dB}$, Corrected Pattern Standard Deviation (CPSD) melhor que $1 \%$ e pelo menos $50 \%$ dos pontos testados dentro dos limites de normalidade; $b$ ) dano moderado: MD entre $-6 \mathrm{~dB}$ e $-15 \mathrm{~dB}$ e CPSD pior que $1 \%$; c) dano avançado: MD pior que $-15 \mathrm{~dB}$ ou CPSD pior que $1 \%$.

Para interpretação do exame do TOPSS, três imagens consecutivas e independentes são obtidas, e uma imagem média é criada a partir das imagens individuais. O uso das três imagens no TOPSS é mandatório, e não opcional como no caso do GDx. Para ser incluída, cada imagem deveria passar pelos critérios de qualidade da máquina. Não houve dilatação pupilar nos pacientes examinados e as luzes do ambiente foram mantidas acesas. Todas as aquisições foram realizadas por um mesmo examinador (LM), que delineou as margens do disco óptico de cada paciente. O TOPSS apresenta 12 parâmetros dispostos a seguir: average disc diameter, total disc area, cup area, cup shape, cup volume, average cup depth, average disc depth, neuroretinal rim (NRR) volume, NRR area, cup/disc area ratio, horizontal cup/disc ratio e vertical cup/disc ratio.

A análise estatística foi realizada utilizando-se o software SPSS, versão 10,1 (SPSS Inc, Chicago, IL). O teste de Komogorov-Smirnov foi utilizado para testar a distribuição das amostras. As correlações entre os índices do campo visual (MD e CPSD) e os 12 parâmetros do TOPSS foram obtidas utilizandose a Correlação de Spearman (r), que mede a associação entre duas variáveis de distribuição não Gaussiana. A estatística descritiva foi realizada calculando-se a média e o desvio padrão para cada parâmetro do TOPSS, e a diferença entre indivíduos normais e pacientes glaucomatosos obtida utilizando-se o Teste de T de Student Independente para variáveis com distribuição normal e o Teste U de Mann-Whitney quando os valores assumiram disposição não Gaussiana. Para dados categóricos foi utilizado o Teste de Qui-Quadrado ou o Teste 
Exato de Fisher. No nosso trabalho, valores de p abaixo de 0,016 foram considerados significativos após correção do erro alfa ${ }^{(22)}$.

\section{RESULTADOS}

Um total de 200 indivíduos foram incluídos no estudo, 88 normais e 112 glaucomatosos. A idade média no grupo glaucomatoso $(63,6 \pm 13,11$ anos) foi significativamente maior que nos indivíduos normais $(47,32 \pm 6,03$ anos $)(\mathrm{p}<0,001)$. Em relação à severidade da lesão glaucomatosa, $36(32,1 \%)$ pacientes foram classificados como glaucoma inicial, $47(42,0 \%)$ como moderado e $29(25,9 \%)$ como glaucoma avançado. O MD médio da população glaucomatosa incluída foi de $-10,63 \pm 7,58$ dB. Os dados demográficos estão ilustrados na tabela 1 .

Ao se compararem os parâmetros do TOPSS e de campo visual para ambos os grupos, verificamos diferença estatisticamente significante para total disc area, cup area, cup volume, cup shape, average disc depth, cup/disc area ratio, horizontal cup/disc ratio, vertical cup/disc ratio, MD, CPSD ( $p<0,001)$, average disc diameter $(\mathrm{p}=0,001)$ e NRR volume $(\mathrm{p}=0,003)$. Average cup depth e NRR area não apresentaram diferença estatisticamente significante entre os grupos $(\mathrm{p}=0,56 \mathrm{e} p=0,50$, respectivamente) (Tabela 2).

Os parâmetros do TOPSS que apresentaram as correlações mais significativas com os índices de campo visual (MD e CPSD) foram: Cup Area (MD: $r=-0,538, p<0,001$; CPSD: $r=$ $0,512, \mathrm{p}<0,001)$, Vertical C/D ratio (MD: $\mathrm{r}=-0,506, \mathrm{p}<0,001$; CPSD: $r=-0,483, p<0,001)$ e Cup/Disc Area Ratio (MD: $r=$ $-0,458, p<0,001$; CPSD: $r=0,453, p<0,001)$. A tabela 3 revela a correlação entre os 12 parâmetros do TOPSS e os índices do Campo Visual. As figuras 1 e 2 ilustram a distribuição nos indivíduos normais e glaucomatosos dos 2 parâmetros do TOPSS (Cup Area e Vertical C/D ratio) com correlação mais significativa com MD e CPSD.

\section{DISCUSSÃ O}

Muito tem-se estudado sobre novos métodos para diagnóstico precoce do glaucoma. Entretanto, o campo visual ainda é considerado como método definitivo para se classificar o paciente como glaucomatoso. Entretando, as variabilidades existentes entre os exames (long-term fluctuation) e durante o mesmo (short-term fluctuation), especialmente nos pontos próximos a escotomas pré-existentes ${ }^{(23)}$, aliado à fadiga causada pelo teste ${ }^{(24)}$, limitam a sua utilidade. Um outro ponto crucial refere-se ao fato de que o defeito perimétrico apenas ocorre após uma substancial perda de células ganglionares retinianas ${ }^{(1-2)}$. Para se testar a possível utilidade de um novo

\begin{tabular}{|c|c|c|c|}
\hline \multicolumn{4}{|c|}{ Tabela 1. Dados demográficos } \\
\hline & Normais & Glaucoma & $\mathbf{p}$ \\
\hline Número (n) & 88 & 112 & \\
\hline Idade $(\text { anos })^{*}$ & 47 & 63 & $<0,001$ \\
\hline Raça (branca/negra/asiática)" & $62 / 24 / 02$ & $74 / 34 / 04$ & 0,75 \\
\hline Sexo (masculino/feminino) $)^{* *}$ & $35 / 53$ & $56 / 56$ & 0,14 \\
\hline Equivalente esférico (dioptrias)\#\# & $0,32 \pm 0,92$ & $-0,3 \pm 2,34$ & 0,01 \\
\hline Olho (direito/esquerdo)** & $49 / 39$ & $64 / 48$ & 0,95 \\
\hline
\end{tabular}

Tabela 2. Parâmetros do TOPSS e Campo Visual em olhos normais e glaucomatosos

\begin{tabular}{|c|c|c|c|}
\hline & Normais & Glaucoma & $\mathbf{p}^{*}$ \\
\hline Average Disc Diameter (mm) & $1,68 \pm 0,19$ & $1,92 \pm 0,22$ & $<0,001$ \\
\hline Total Disc Area $\left(\mathrm{mm}^{2}\right)$ & $2,22 \pm 0,55$ & $2,82 \pm 0,64$ & $<0,001$ \\
\hline Cup Area $\left(\mathrm{mm}^{2}\right)$ & $0,84 \pm 0,46$ & $1,50 \pm 0,68$ & $<0,001$ \\
\hline Cup Shape & $-0,73 \pm 0,77$ & $-1,07 \pm 0,80$ & $<0,001$ \\
\hline Cup Volume $\left(\mathrm{mm}^{3}\right)$ & $-0,24 \pm 0,19$ & $-0,46 \pm 0,38$ & $<0,001$ \\
\hline Average Cup Depth (mm) & $-0,26 \pm 0,15$ & $-0,28 \pm 0,14$ & 0,56 \\
\hline Average Disc Depth (mm) & $0,053 \pm 0,14$ & $-0,066 \pm 0,16$ & 0,001 \\
\hline NRR Volume $\left(\mathrm{mm}^{3}\right)$ & $0,35 \pm 0,22$ & $0,26 \pm 0,15$ & 0,003 \\
\hline NRR Area $\left(\mathrm{mm}^{2}\right)$ & $1,38 \pm 0,45$ & $1,32 \pm 0,55$ & 0,50 \\
\hline Cup/Disc Area Ratio & $0,37 \pm 0,16$ & $0,52 \pm 0,19$ & $<0,001$ \\
\hline Horizontal C/D Ratio & $0,59 \pm 0,19$ & $0,69 \pm 0,20$ & $<0,001$ \\
\hline Vertical C/D Ratio & $0,45 \pm 0,21$ & $0,65 \pm 0,20$ & $<0,001$ \\
\hline Mean Deviation (dB) & $-0,84 \pm 1,26$ & $-10,63 \pm 7,58$ & $<0,001$ \\
\hline Corrected Pattern Standard Deviation (dB) & $1,59 \pm 0,99$ & $5,50 \pm 3,09$ & $<0,001$ \\
\hline
\end{tabular}



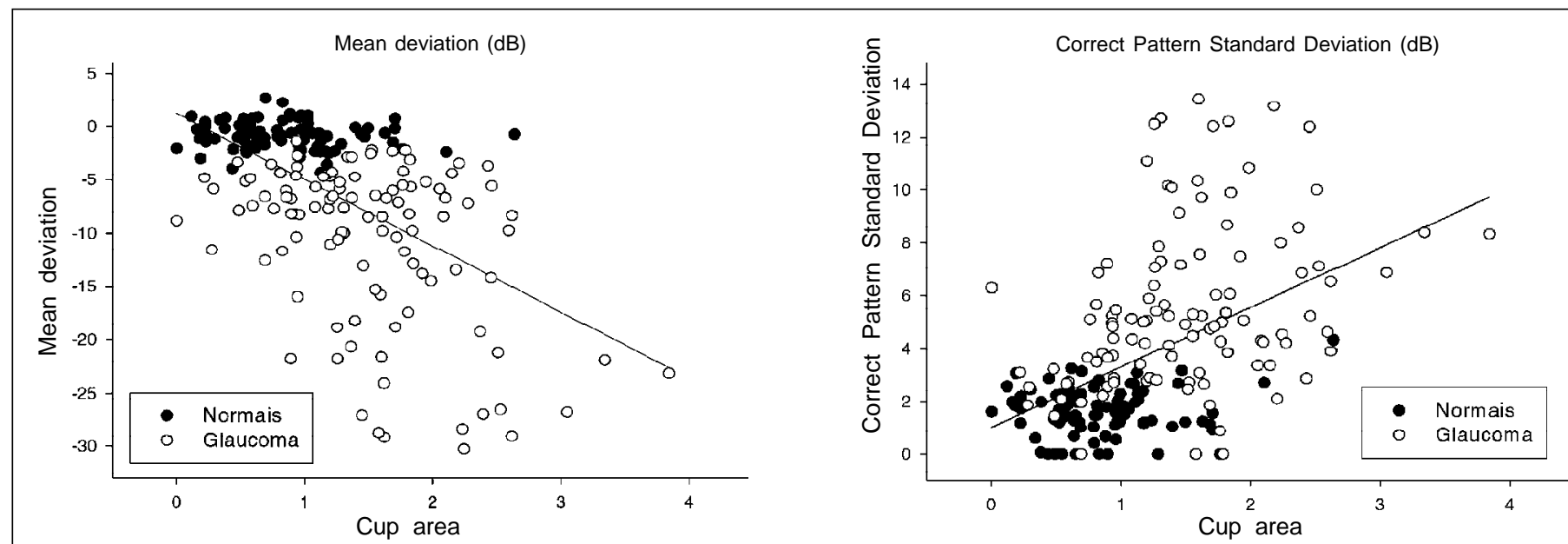

Figura 1 - Correlação entre Cup Area e índices do campo visual
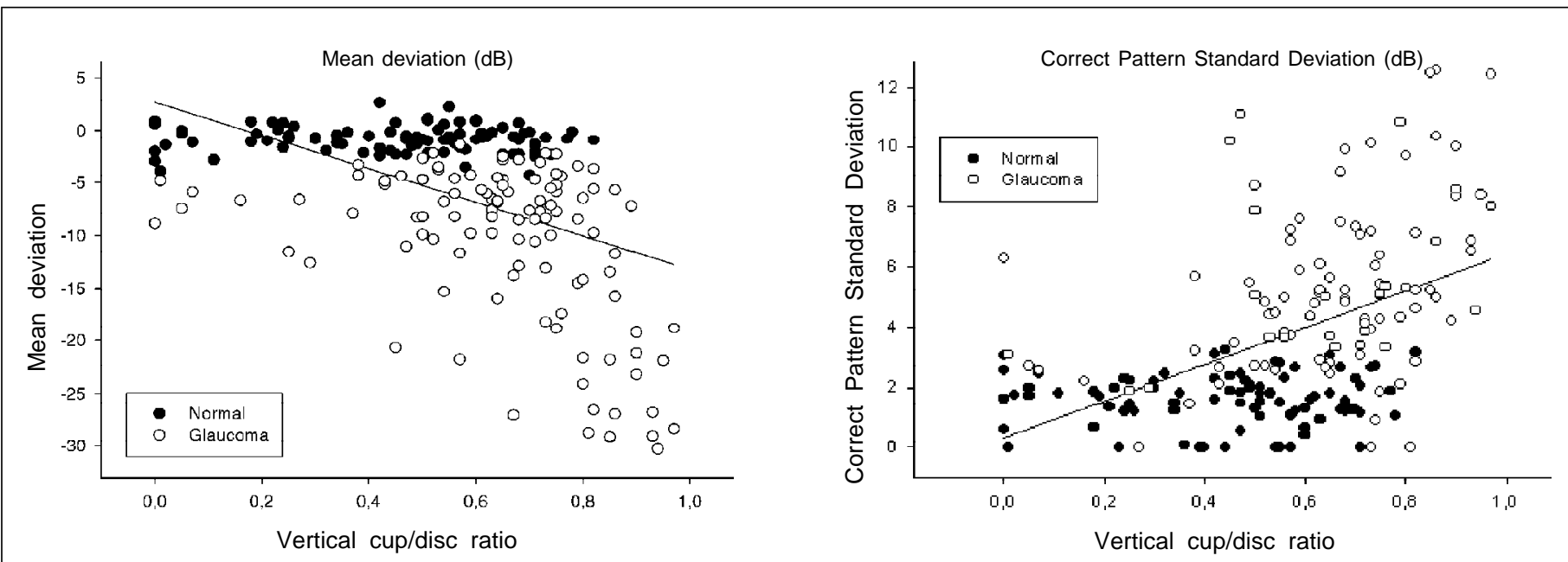

Figura 2 - Correlação entre Vertical Cup/Disc Ratio e índices do campo visual

método diagnóstico, é fundamental correlacioná-lo ao "gold standard" que avalia a perda funcional que ocorre no glaucoma (defeito de campo visual).

Em relação à topografia de disco óptico, estudos anteriores testaram a correlação entre o HRT e índices do campo visual. Em um estudo com 46 pacientes com glaucoma inicial ou moderado ${ }^{(16)}$ (MD: $-4,8 \pm 6,2 \mathrm{~dB}$ ), foi verificado que o parâmetro Cup Shape Measure foi o único que apresentou forte correlação com MD (r= -0,65, p<0,001) e CPSD (r=0,55, $\mathrm{p}<0,001)$. Entretanto, em outro trabalho ${ }^{(17)}$, foi constatado que o parâmetro com a correlação mais significativa foi Rim Area (MD: $r=0,44, p<0,001$; CPSD: $r=-0,48, p<0,001$ ) ao se estudar uma amostra mesclada com indivíduos normais $(n=59)$, hipertensos oculares $(n=64)$, glaucoma de pressão intra-ocular dentro da média $(n=47)$ e glaucoma com pressão intra-ocular acima da média $(n=124)$. É importante salientar que os autores consideraram uma correlação estatisticamente significante entre o campo visual e vários parâmetros derivados do HRT apenas baseados nos valores de $\mathrm{p}$, quando, na verdade, os valores do coeficiente de Pearson indicaram uma fraca relação da maioria desses parâmetros citados (Cup Area: MD: $r=-0,28$, CPSD: $r=$ 0,25; C/D Area Ratio: MD: $r=-0,25$, CPSD: $r=0,24$; RNFL cross-sectional area: MD: $r=0,27$, CPSD: $r=-0,34)^{(17)}$. Em artigo recente, ao se realizar análise topográfica global e setorial, foi verificado que os parâmetros do topógrafo de disco óptico que melhor se correlacionaram com o MD foram: global average slope $(r=-0,60, p<0,001)$ e cup/disc ratio $(r=-0,40$, $\mathrm{p}<0,001)$, sendo o último valor semelhante ao encontrado em nosso estudo (Tabela 3 ).

Embora exista uma diferença altamente significativa entre os grupos estudados para praticamente todos os parâmetros do TOPSS (Tabela 2), a correlação entre os mesmos, MD e CPSD foi, na média, apenas moderada (Tabela 3). No nosso estudo, os parâmetros do TOPSS com melhor correlação com 


\begin{tabular}{|lcrrr|}
\hline & Tabela 3. Correlação entre os parâmetros do TOPSS e índices do Campo Visual & \\
Parâmetro & MD $^{*}$ & $\mathbf{p}$ & CPSD $^{*}$ & P \\
Cup Area & $-0,538$ & $<0,001$ & 0,512 & $<0,001$ \\
C/D Area Ratio & $-0,458$ & $<0,001$ & 0,453 & $<0,001$ \\
Avg. Disc Depth & 0,416 & $<0,001$ & $-0,412$ & $<0,001$ \\
Vertical C/D Ratio & $-0,506$ & $<0,001$ & 0,483 & $<0,001$ \\
Cup Volume & 0,376 & $<0,001$ & $-0,398$ & $<0,001$ \\
NRR Volume & 0,329 & $<0,001$ & $-0,309$ & $<0,001$ \\
Avg. Disc Diameter & $-0,491$ & $<0,001$ & 0,395 & 0,001 \\
NRR Area & 0,181 & 0,01 & $-0,206$ & $<0,001$ \\
Horizontal C/D Ratio & $-0,302$ & $<0,001$ & 0,322 & $<0,001$ \\
Total Disc Area & $-0,462$ & $<0,001$ & 0,002 \\
Cup Shape & 0,217 & 0,002 & $-0,367$ & 0,07 \\
Avg. Cup Depth & 0,054 & 0,45 & $-0,130$ & \\
"Correlação de Spearman & & & & \\
\hline
\end{tabular}

os índices do campo visual foram Cup Area (MD: $r=-0,538$; CPSD: $r=0,512$ ) Vertical C/D Ratio (MD: $r=-0,506$, CPSD: $r=$ $0,483)$. Entretanto, podemos verificar importante variabilidade desses parâmetros em diferentes níveis de dano glaucomatoso, representados pelo MD e CPSD. Em outras palavras, para um mesmo valor de MD (ou CPSD), constatamos uma variação grande nos valores estruturais (Figuras 1 e 2). Esse achado condiz com estudos prévios, onde provou-se que escavação aumentada como dado isolado não é patognomônico de glaucoma, e portanto pode existir tanto em olhos normais como glaucomatosos, embora com maior incidência no último gru$\mathrm{po}^{(25)}$. Esse dado vem reforçar a premissa da grande variabilidade individual das estruturas do disco óptico, e consequente necessidade do uso comitante de dois ou mais parâmetros topográficos para melhorar a capacidade de diagnóstico desses instrumentos ${ }^{(9,26)}$.

Outro dado advindo do nosso estudo é que, apesar do HRT e TOPSS serem ambos sistemas confocais de aquisição da imagem topográfica do disco óptico, comportam-se de maneira diferente com referência às suas variáveis individuais. No estudo em que o parâmetro Rim Area teve a melhor correlação com o campo visual ${ }^{(17)}$, as variáveis Cup Area e C/D Area Ratio foram fracamente correlacionadas com MD e CPSD. Esses resultados são praticamente opostos aos encontrados na nossa amostra, onde Cup Area e Vertical C/D Ratio foram os parâmetros com melhor correlação com o campo visual, enquanto o correspondente parâmetro do TOPSS para Rim Area (NRR Area) apresentou valores de r borderline ou não significativos (tabela 3 ).

\section{CONCLUSÃ O}

Concluindo, as variáveis do TOPSS apresentam, no geral, correlação satisfatória com os índices do campo visual. Apesar de alguns desses parâmetros apresentarem correlação altamente significativa, existe grande variabilidade dos parâmetros estruturais para um mesmo nível de dano funcional, limi- tando a utilização isolada de parâmetros estruturais derivados do TOPSS no diagnóstico do glaucoma.

\section{A BSTR ACT}

Purpose: To evaluate the correlation between the parameters derived from the topographic scanning system (TOPSS) and visual field indices. Methods: One-hundred and twelve primary open-angle glaucoma patients and 88 normal controls were enrolled in the study. All individuals underwent a thorough ophthalmic examination, including visual field (Humphrey 24-2, Full Threshold mode), evaluating Mean Deviation (MD) and correct pattern standard deviation (CPSD) and TOPSS (average disc diameter, total disc area, cup area, cup shape, cup volume, average cup depth, average disc depth, neuroretinal rim (NRR) volume, NRR area, cup/disc area ratio, horizontal cup/disc ratio e vertical cup/disc ratio). The comparison between groups was done using the Mann-Whitney Test or the independent Student's t test. The correlation between the visual field indices and TOPSS parameters was performed with the Spearman's correlation. Results: The TOPSS parameters with better correlation were: cup area (MD: $\mathrm{r}=-0.538, \mathrm{p}<0.001$; CPSD: $\mathrm{r}=0.512, \mathrm{p}<0.001)$, vertical C/D ratio (MD: $\mathrm{r}=-0.506, \mathrm{p}<0.001$; CPSD: $\mathrm{r}=0.483, \mathrm{p}<0.001$ ) e cup/disc area ratio (MD: $r=-0.458, p<0.001$; CPSD: $r=0.453$, $p<0.001$ ). However, there was considerable variability between groups with different levels of visual field loss. Conclusions: The TOPSS variables presented, in general, substantial correlation with visual field indices. Although some of these parameters showed highly significant correlations, considerable variability of the structural parameters for the same level of functional damage may limit the usefulness of TOPSS isolated parameters in the diagnosis of glaucoma.

Keywords: Glaucoma/diagnosis; Optic disc/pathology; Perimetry; Diagnostic techniques, ophthalmological 


\section{REFERÊNCIAS}

1. Kerrigan-Baumrind LA, Quigley HA, Pease ME, Kerrigan DF, Mitchell RS Number of ganglion cells in glaucoma eyes compared with threshold visual field tests in the same persons. Invest Ophthalmol Vis Sci 2000;41:741-8.

2. Quigley HA, Addicks EM, Green WR. Optic nerve damage in human glaucoma. III. Quantitative correlation of nerve fiber loss and visual field defect in glaucoma, ischemic neuropathy, papilledema, and toxic neuropathy. Arch Ophthalmol 1982;100:135-46.

3. Sommer A, Pollack I, Maumenee AE. Optic disc parameters and onset of glaucomatous field loss. I. Methods and progressive changes in disc morphology. Arch Ophthalmol 1979;97:1444-8.

4. Quigley HA, Katz J, Derick RJ, Gilbert D, Sommer A. An evaluation of optic disc and nerve fiber layer examinations in monitoring progression of early glaucoma damage. Ophthalmology 1992;99:19-28.

5. Sommer A, Katz J, Quigley HA, Miller NR, Robin AL, Richter RC, Witt KA. Clinically detectable nerve fiber atrophy precedes the onset of glaucomatous field loss. Arch Ophthalmol 1991;109:77-83.

6. Weinreb RN, Shakiba S, Zangwill L. Scanning laser polarimetry to measure the nerve fiber layer of normal and glaucomatous eyes. Am J Ophthalmol 1995; 119:627-36.

7. Weinreb RN, Zangwill L, Berry CC, Bathija R, Sample PA. Detection of glaucoma with scanning laser polarimetry. Arch Ophthalmol 1998;116:1583-9.

8. Wollstein G, Garway-Heath DF, Hitchings RA. Identification of early glaucoma cases with the scanning laser ophthalmoscope. Ophthalmology 1998; 105:1557-63.

9. Mikelberg FS, Parfitt CM, Swindale NV, Graham SL, Drance SM, Gosine R. Ability of the Heidelberg Retina Tomograph to detect early glaucomatous visual field loss. J Glaucoma 1995;4:242-7.

10. Ahn BS, Kee C. Ability of a confocal scanning laser ophthalmoscope (TopSS) to detect early glaucomatous visual field defect. Br J Ophthalmol 2000;84:852-5.

11. Geyer O, Michaeli-Cohen A, Silver DM, Versano D, Neudorfer M, Dzhanov R, Lazar M. Reproducibility of topographic measures of the glaucomatous optic nerve head. Br J Ophthalmol 1998;82:14-7.

12. Weinreb RN, Shakiba S, Sample PA, Shahrokni, S, van Horn S, Garden VS et al. Association between quantitative nerve fiber layer measurement and visual field loss in glaucoma. Am J Ophthalmol 1995;120:732-8.
13. Almeida PB, Almeida GV, Cohen R, Prata Júnior JA, Melo PAA. Correlação e correspondência topográfica entre espessura da camada de fibras nervosas da retina e campo visual no glaucoma primário de ângulo aberto. Arq Bras Oftalmol 2001;64:109-15.

14. Cullinane AB, Waldock A, Diamond JP, Sparrow JM. Optic disc cup slope and visual field indices in normal, ocular hypertensive and early glaucomatous eyes. Br J Ophthalmol 2002;86:555-9.

15. Sturmer J, Bernasconi P, Caubergh MJ, Frei C, Yanar A, Gloor B. Value of scanning laser ophthalmoscopy and polarimetry compared with perimetry in evaluating glaucomatous changes in the optic papilla and nerve fiber layer. Ophthalmologe 1996;93:520-6.

16. Brigatti L, Caprioli J. Correlation of visual field with scanning confocal laser optic disc measurements in glaucoma. Arch Ophthalmol 1995;113:1191-4.

17. Iester M, Mikelberg FS, Courtright P, Drance SM. Correlation between the visual field indices and Heidelberg retina tomograph parameters. J Glaucoma 1997;6:78-82.

18. Mikelberg FS, Wijsman K, Schulzer M. Reproducibility of the Topographic Parameters obtained with the Heidelberg Retina Tomograph. J Glaucoma 1993;2:101-3.

19. Anderson. DR, Patella VM. Automated Static Perimetry. $2^{\text {nd }}$ ed. St. Louis: Missouri, USA; 1999.

20. Chylack LT Jr., Wolfe JK, Singer DM, Leske MC, Bullimore MA, Bailey IL et al. The lens opacities classification system III. The Longitudinal Study of Cataract Study Group. Arch Ophthalmol 1993;111:831-6.

21. Hodapp E, Parrish II RK, Anderson DR. Clinical decisions in glaucoma. St. Louis: Missouri, USA; 1993.

22. Cross EM, Chaffin WW. Use of the binomial theorem in interpreting results of multiple tests of significance. Educ Psychol Meas 1982;42:25-34.

23. Heijl A, Drance SM. Changes in differential threshold in patients with glaucoma during prolonged perimetry. Br J Ophthalmol 1983;67:512-6.

24. Hudson C, Wild JM, O'Neill EC. Fatigue effects during a single session of automated static threshold perimetry. Invest Ophthalmol Vis Sci 1994; 35:268-80.

25. Jonas JB, Zach FM, Gusek GC, Naumann GO. Pseudoglaucomatous physiologic large cups. Am J Ophthalmol 1989;107:137-44.

26. Caprioli J. Discrimination between normal and glaucomatous eyes. Invest Ophthalmol Vis Sci 1992;33:153-9. 